\title{
Is the World Objective?
}

\author{
Shantena Augusto Sabbadini \\ Pari Center for New Learning, Pari, Italy \\ Email: info@shantena.com
}

Received 25 February 2014; revised 25 March 2014; accepted 1 April 2014

Copyright (C) 2014 by author and Scientific Research Publishing Inc.

This work is licensed under the Creative Commons Attribution International License (CC BY). http://creativecommons.org/licenses/by/4.0/

(c) (i) Open Access

\section{Abstract}

The assumption of an objective world underlying all our experiences is deeply embedded in our everyday thinking and in our language, and has been an unquestioned presupposition of the scientific enterprise for at least four centuries. Yet it is well known that quantum physics raises some highly problematic questions in this respect, questions that are still open, almost a century from the inception of the theory. I would like to propose that these questions stem from deep philosophical assumptions and can be answered in a much more coherent way if we understand the notion of an objective world not as a primary reality, but as an emergent aspect of a primary process that can be described as "experiencing". A coherent description of the appearance of an objective world in the process of experiencing relies on a certain understanding of what is technically known as the "quantum measurement problem", i.e. the description of the process of observation in quantum physics, which is the key problematic aspect of the theory. I will propose that the basic features that characterize a quantum observation have a much wider scope, and describe in fact all acquisition of information by an "embodied observer", in short all experiencing happening in the world. By applying to this larger context work I have done in the more technical frame of the quantum measurement problem it can be shown that the nature of the process of experiencing is such as to inevitably generate the appearance of an objective world. Thus the objectivity of the world, although not primary and ultimately real, is an intrinsic consequence of our "experiential embodiment", i.e. of the fact that we are in the world and all our experiences correspond to physical happenings in the world. When examined in this perspective, the "paradoxes" of quantum physics are an invitation to abandon the metaphysical assumption of the primacy of the objective dimension of reality. A philosophical position much more in tune with quantum physics views the process of experiencing as the primary source of both subject and object, consciousness and world.

\section{Keywords}

Subject; Object; Objective World; Quantum Physics; Quantum Measurement; Von Neumann Chain; Observer; Embodiment; Consciousness; Persistence of Information; Quantum Eraser 


\section{Introduction}

The assumption of an objective world underlying all our experiences is deeply embedded in our everyday thinking and in our language, and has been an unquestioned presupposition of the scientific enterprise for at least four centuries.

Yet it is well known that quantum physics raises some highly problematic questions in this respect, questions that are still open, almost a century from the inception of the theory. I would like to propose that these questions stem from deep philosophical assumptions and can be answered in a much more coherent way if we understand the notion of an objective world not as a primary reality, but as an emergent aspect of a primary process that can be described as "experiencing”.

A coherent description of the appearance of an objective world in the process of experiencing relies on a certain understanding of what is technically known as the "quantum measurement problem", i.e. the description of the process of observation in quantum physics, which is the key problematic aspect of the theory. I will propose that the basic features that characterize a quantum observation have a much wider scope, and describe in fact all acquisition of information by an "embodied observer", in short all experiencing happening in the world.

By applying to this larger context work I have done in the more technical frame of the quantum measurement problem (the "persistence of information" approach, Sabbadini, 2006) ${ }^{1}$ it can be shown that the nature of the process of experiencing is such as to inevitably generate the appearance of an objective world. Thus the objectivity of the world, although not primary and ultimately real, is an intrinsic consequence of our "experiential embodiment”, i.e. of the fact that we are in the world and all our experiences correspond to physical happenings in the world.

If we do not a priori assume the existence of an objective world, the notion of a physical happening needs to be defined in purely quantum terms, and in fact such a definition is an essential ingredient of the persistence of information approach. Likewise, a crucial requirement of any theory that does not assume the objectivity of the world as a primary given is that it should account for the consistence of the world experienced by different observers, an issue known in the context of the quantum measurement problem as "Wigner's friend". The persistence of information approach does indeed guarantee such a consistence ${ }^{2}$. Therefore, in spite of the multiplicity of experiencing subjects, we all share the appearance of the same objective world.

This consistence "confirms" the objectivity of the world in our daily interactions with each other. We ordinarily assume that we are objective beings moving in an objective world, and we only need to question such an assumption when we deal with those peculiar experiences that are quantum experiments. The persistence of information approach shows that, whether or not an objective world independent from subjectivity or consciousness exists, the world is bound to appear that way to an embodied observer for a fundamental reason, having to do with the nature of the process of experiencing itself.

When examined in this perspective, the "paradoxes" of quantum physics are an invitation to abandon the metaphysical assumption of the primacy of the objective dimension of reality. A philosophical position much more in tune with quantum physics views the process of experiencing as the primary source of both subject and object, consciousness and world.

\section{The Quantum Measurement Problem}

In spite of the enormous success of quantum physics, almost a century after its inception some fundamental issues at the core of the theory remain controversial and are still incompletely understood. The crucial point is the quantum description of the process of observation, the so-called "quantum measurement problem". The essence of the problem can be described in the following terms.

A central feature of quantum physics is the radically novel notion of superposition of states. Unlike in classical physics, where the state of a system fully defines all of its properties, in quantum physics there is an intrinsic limit to the amount of information the state of a system contains. In any state there are always observables whose value remains undefined, and the state of the system can then be represented as a superposition of eigenstates of such an observable ${ }^{3}$. Loosely speaking, the system is "suspended between various potentialities" with respect to the value of that observable, and one of these potentialities gets actualized in an unpredictable

\footnotetext{
${ }^{1}$ This paper can also be downloaded from the author's website, http://www.shantena.com/en/physicslectures/quantummeasurement/.

${ }^{2}$ The proof of this statement is simple, but too technical for the context of the present article. It can be deduced by applying the reasoning of the "persistence of information" paper cited in footnote 1 to a multiple observers situation.

${ }^{3}$ An eigenstate of an observable is a state in which the observable has a well defined value.
} 
way when a measurement of the observable is carried out. Providing a coherent description of that actualizing is the challenge of the quantum measurement problem.

Let us look at this in greater detail. I apologize for using symbols here: I am afraid that this is necessary for clarity. Let us say that we have an apparatus $\mathbf{M}$ for measuring the property $\mathrm{P}$ of the object system $\mathbf{S}^{4}$. What defines $\mathbf{M}$ as an apparatus for measuring $\mathbf{P}$ in $\mathbf{S}$ is that the interaction of $\mathbf{M}$ with $\mathbf{S}$ causes a property $\mathbf{M}$ of $\mathbf{M}$ to assume, at end of the measuring process, a value correlated with the value initially possessed by the property $\mathrm{P}$ of S. E.g., if initially $\mathbf{P}=0$ in $\mathbf{S}$, then at the end $\mathbf{M}=0$ in $\mathbf{M}$; if initially $\mathbf{P}=1 \mathrm{in} \mathbf{S}$, then at the end $\mathbf{M}=1$ in $\mathbf{M}$. The property $\mathbf{M}$ of $\mathbf{M}$ can be thought of as an abstract two-valued "pointer position" indicating the result of the measurement of $\mathrm{P}$ as 1 or 0 .

What happens then if the state of the quantum system $\mathbf{S}$ is a superposition of eigenstates of the property $\mathrm{P}$, i.e. a superposition of a state in which $\mathrm{P}=0$ and a state in which $\mathrm{P}=1$ ? If we apply to the total system $\mathbf{S}+\mathbf{M}$ (object system and apparatus) the ordinary time evolution formalism of quantum physics (Schrödinger's equation or an appropriate generalization thereof) we find that after the interaction the system $\mathbf{S}+\mathbf{M}$ finds itself in a superposition of states corresponding to the two possible results of the measurement, i.e. in a superposition of a state in which $P=0$ and $M=0$ and a state in which $P=1$ and $M=1$. Quantum physics deals essentially with statistics, therefore we can think of measuring $\mathbf{P}$ on many identical copies of the system $\mathbf{S}$. We will end up with a collection of systems $\mathbf{S}+\mathbf{M}$ all in the state described above, a superposition of a state in which $\mathrm{P}=0$ and $\mathbf{M}=0$ and a state in which $\mathrm{P}=1$ and $\mathrm{M}=1$.

This is quite a different state of affairs from what happens in a classical measurement and from what is actually observed.

Let us consider for a moment what happens in a classical measurement. Suppose that $\mathrm{P}$ is a property of a classical system $\mathbf{S}$ about which we have incomplete information, so that a measurement of $\mathrm{P}$ can give the result $\mathrm{P}=$ 0 or the result $P=1$. Statistically such a situation is represented by a collection of identical systems, a fraction of which is in a state such that $\mathrm{P}=0$ and another fraction is in a state such that $\mathrm{P}=1$. By measuring the property $\mathrm{P}$ with the measuring apparatus $\mathbf{M}$ we end up with a collection of systems $\mathbf{S}+\mathbf{M}$, a fraction of which is in a state such that $\mathrm{P}=0$ and $\mathrm{M}=0$ and another fraction is in a state such that $\mathrm{P}=1$ and $\mathrm{M}=1$.

How are the quantum and the classical statistical situations different? The difference becomes apparent as soon as we look at the predictions they give for subsequent measurements performed on the total system $\mathbf{S}+\mathbf{M}$. A general observation on $\mathbf{S}+\mathbf{M}$ can be described as the "product" $\mathrm{Q} \otimes \mathrm{R}$ of a general property $\mathrm{Q}$ of the system $\mathbf{S}$ and a general property $\mathrm{R}$ of the system $\mathbf{M}$. The expectation value of such a product on the classical collection of systems has the form:

$$
\langle\mathrm{Q} \otimes \mathrm{R}\rangle_{\text {classical }}=\langle\mathrm{Q} \otimes \mathrm{R}\rangle_{0}+\langle\mathrm{Q} \otimes \mathrm{R}\rangle_{1},
$$

where the first term is the contribution of the fraction of systems that is in a state such that $\mathrm{P}=0$ and $\mathrm{M}=0$ and the second term is the contribution of the fraction of systems that is in a state such that $\mathrm{P}=1$ and $\mathrm{M}=1$. But no such neat separation happens in the quantum case, since in the quantum collection each system is in a superposition of both states. The expectation value of the product $\mathrm{Q} \otimes \mathrm{R}$ on the quantum collection of systems has the form:

$$
\langle\mathrm{Q} \otimes \mathrm{R}\rangle_{\text {quantum }}=\langle\mathrm{Q} \otimes \mathrm{R}\rangle_{0}+\langle\mathrm{Q} \otimes \mathrm{R}\rangle_{1}+\langle\mathrm{Q} \otimes \mathrm{R}\rangle_{01}+\langle\mathrm{Q} \otimes \mathrm{R}\rangle_{10}
$$

where the last two terms, called "interference terms", represent a joint contribution of the state in which $\mathrm{P}=0$ and $\mathrm{M}=0$ and the state in which that $\mathrm{P}=1$ and $\mathrm{M}=1^{5}$.

The above discussion can be summarized by saying that the application of the quantum time evolution formalism to a superposition of states inevitably leads to another superposition of states. It never leads to a collection of systems that can be divided into subsets corresponding to a specific result of the measurement (in statistical jargon known as a "mixed ensemble" or simply a "mixture").

This result appears paradoxical, since it does not correspond to actual experience. In actuality, when we re-

\footnotetext{
${ }^{4} \mathrm{~A}$ property is a two-valued observable, whose values can be thought of as yes/no, true/false, $1 / 0$, etc. (the system either possesses that property or it does not). In order to keep the discussion as simple as possible, in the following I will consider properties of the various systems in question, rather than general observables. By dividing the spectrum of values of a general observable A into finite intervals and asking questions of the type "does the value of A fall into this specific interval or not?", a general observable can always be reduced to a collection of properties. There is therefore no loss of generality in confining our discussion to properties. In mathematical terms a property corresponds to a projector operator in the Hilbert space of the states of the system.

${ }^{5}$ The name "interference terms" comes from the analogy with optics, where we encounter such an effect when two coherent waves coming from different sources meet, generating an oscillatory pattern.
} 
peatedly perform a measurement of the property $\mathrm{P}$ on a collection of identical quantum systems $\mathbf{S}$, the outcome is indeed a mixed ensemble, in which a fraction is in the state corresponding to $\mathrm{P}=0$ and $\mathrm{M}=0$ and another fraction is in the state corresponding to $\mathrm{P}=1$ and $\mathrm{M}=1$. How to explain the discrepancy between the results predicted by the quantum time evolution formalism and actual experience is the essence of the quantum measurement problem.

When the problem is formulated in these terms, one would be tempted to conclude that the mistake resides in the quantum time evolution formalism. That is of course a possibility, but not one to be considered lightly. The equations governing the time evolution of quantum systems have so far proved extremely accurate in all the situations in which they have been put to the test. And the discrepancy comes from a basic characteristic of those equations, namely their linearity, so that the necessary amendment would be no small modification. The challenge, if we should try to pursue such an avenue, would be altering the basic structure of quantum physics in a way that does away with linearity, while still preserving the explanatory capabilities of the present theory. No convincing theory along these lines has been proposed so far.

\section{Quantum Measurement as a Pattern for Acquisition of Information in General}

Before proceeding further in our examination of the quantum measurement problem and of the various proposed solutions, it is worth enlarging our perspective and looking at what is really implied. The quantum measurement problem is not merely a technical problem, as it may appear at first sight. What is at stake is not simply how we explain certain anomalies that show up in physics laboratories. It involves our representation of reality. Is the world classical or quantum? Is it or is it not endowed with objective properties? What, if any, is the role of consciousness in it?

The way we model a quantum measurement process actually has a much wider scope, it can be viewed as the general pattern of all perception, i.e. the general pattern of all acquisition of information by an embodied observer. The basic model of a quantum measurement process was first outlined by John von Neumann (von Neumann, 1932). It consists of a chain of interacting systems, starting with the observed quantum system $\mathbf{S}$ and ending with the measuring apparatus $\mathbf{M}$, through a series of intermediate steps $\mathbf{M}_{1}, \mathbf{M}_{2}$, etc., which correspond to an amplification process going from the microscopic to the macroscopic. The interactions along the chain generate a correlation between the state of $\mathbf{S}$ and the state of $\mathbf{M}_{1}$, then between the state of $\mathbf{M}_{1}$ and the state of $\mathbf{M}_{2}$, etc., so that at the end the state of the measuring apparatus $\mathbf{M}$ is correlated with the initial state of the object system $\mathbf{S}$. These correlations can be described by means of 'measuring observables' $M_{1}, M_{2}, \ldots, M$ of the systems $\mathbf{M}_{1}, \mathbf{M}_{2}, \ldots, \mathbf{M}$, which assume correlated values (when $\mathrm{M}_{1}$ assumes a certain value $\mathrm{m}_{1}, \mathbf{M}_{2}$ assumes a corresponding value $\mathrm{m}_{2}$, etc.). This pattern, which is known as a von Neumann chain, is quite general and can be applied to describe any process of acquisition of information.

Consider for example a simple visual perception, say the perception of a red surface. A schematic outline of the process is as follows. Light impinges on the surface and is partly absorbed and partly reflected. If the surface is what we call red, it absorbs mainly the blue components of light and reflects the red components (electromagnetic radiation with a wavelength in the range $620-740 \mathrm{~nm}$ ). The reflected electromagnetic radiation interacts with the lens of my eye that focuses it on the photoreceptive cells of the retina. These respond by producing neural impulses correlated with the wavelength of the impinging radiation. These neural impulses travel up my optic nerve to the lateral geniculate nucleus, which generates corresponding signals delivered through its axons to the primal visual cortex and other places. Here complicated things happen, which I wouldn't presume to be able to describe. But I don't need to, because the point I want to make is simply that in the end a particular firing pattern will take place in my brain, and I will experience the visual sensation that I call "red". The chain of correlating interactions has worked its way up all the way from a characteristic of the surface in front of me (a material that tends to absorb blue wavelengths) to a pattern of firing in my brain (corresponding to the experience of red).

The same is true of all perception, actually of all acquisition of information by an embodied consciousness. By "embodied consciousness" I mean a consciousness whose experiences correspond to physical happenings in a particular "object body" in the world (in the example, my experience of red corresponding to a specific firing pattern in my brain $)^{6}$.

${ }^{6}$ As an important side remark notice that, while all we recognize as consciousness, i.e. as capable of having experiences, is certainly embodied in the above sense, the pattern described is more general and opens up the possibility of enlarging our notion of consciousness beyond what we ordinarily take for granted. We easily recognize consciousness in systems that are similar enough to ourselves. But we might be blind to other forms of embodied consciousness that are very different from our own. 


\section{Approaches to the Quantum Measurement Problem}

The way the discrepancy between the results predicted by the quantum time evolution formalism and actual experience is handled in quantum physics textbooks is usually along the lines of the so-called "Copenhagen interpretation" or "orthodox interpretation" of quantum physics, proposed by Niels Bohr and his school in the 1930's. Such an interpretation assumes that quantum physics is merely an algorithm for calculating connections between classical states. The algorithm includes two different types of time evolution for a quantum system: the ordinary time evolution governed by Schrödinger's equation (or an appropriate generalization thereof), applying to all situations except measurements; and a special time evolution, named "collapse of the state vector" or "collapse of the wave function", which applies to measurement interactions. The collapse of the state vector transforms the quantum superposition that is the outcome of the measurement according to the ordinary time evolution into the corresponding classical mixture.

Such a prescription has a practical value, in that it provides the experimentalist with all she or he needs in order to calculate. But it is hardly a satisfactory solution of the measurement problem. It essentially bypasses the problem by renouncing to include a description of the measuring apparatus in terms of its microscopic components, because, if the measuring apparatus consists of atoms and molecules just like the object system, there is no reason why the interaction of the two should not be described by the ordinary time evolution formalism.

A satisfactory solution of the quantum measurement problem must either offer a mechanism explaining the collapse of the state vector or offer an explanation of the appearance of such a collapse within the frame of the ordinary time evolution formalism of quantum physics.

A variety of solutions along one or the other of these two lines has been proposed since the early 1930's. Only a few representative examples will be briefly described here.

An early approach to the problem postulates that a non-physical interaction between the observer's consciousness and the observed quantum system forces the collapse of the state vector. But this "idealistic" approach opens the way to various paradoxes, well illustrated by the Schrödinger's cat thought experiment (Schrödinger, 1935) and its "Wigner's friend" variation (Wigner, 1967). Not many physicists at present vouch for such a solution.

An equally metaphysical assumption is Everett's many worlds theory (Everett, 1957). It assumes that a quantum measurement causes a "branching" of the universe and each branch of the quantum superposition that is the outcome of the measurement process exists in a separate universe. We are aware of only one result of the measurement, the one that is given in our particular universe; but, unbeknown to us, an exact copy of ourselves exists in each of the other universes, performing the same measurement and obtaining a different result. While not formally confutable, such an assumption seems to radically violate Occam's razor (numquam ponenda est pluralitas sine necessitate).

While both these approaches seem unsatisfactory (but the first is still widely held in lay circles, and the second still has its staunch supporters among physicists), each contains an element worth retaining. Wigner's idealistic approach points to the fact that quantum physics cannot be consistently formulated without mentioning "the observer", i.e. consciousness or subjectivity. Quantum physics does not provide a closed description of an objective world—a statement that will be further discussed in the following.

Everett's many worlds approach points to the fact that, unless we change quantum theory in substantial ways, we are bound to describe the outcome of a measuring process as a superposition of states. Any way to circumvent this requirement amounts essentially to an ad hoc prescription (the classic example being the Copenhagen interpretation).

The most widely shared modern approach to the quantum measurement problem is the so-called "decoherence theory", , which assumes that the outcome of a measurement process is correctly described by a quantum superposition, as predicted by the ordinary time evolution formalism, but argues that all predictions based on such a superposition rapidly become indistinguishable from those based on the corresponding classical mixture. Why is that? Because the interference terms, that make the difference between the predictions of one and the other, rapidly disperse with the increasing size of the systems involved in the von Neumann chain, and quickly become unobservable as the amplification approaches macroscopic proportions. At the end of a measuring process revealing the presence of the interference terms would require performing a detailed observation of the quantum state of the entire measuring apparatus, laboratory and environment (including the experimenter, if present!),

\footnotetext{
${ }^{7}$ See, e.g., (Joos, 2003). The author has contributed to early work in this direction (Daneri, Loinger, \& Prosperi, 1962; Lanz, Prosperi, \& Sabbadini, 1971).
} 
clearly an unfeasible proposition.

Therefore the quantum superposition, which is the true outcome of the measuring process, can be replaced for all practical purposes by the corresponding classical mixture. The difference between the two never rigorously vanishes, but gets progressively lost in the process of amplification and becomes irrelevant on a macroscopic scale. The decoherence answer to the question “why do we perceive a classical world?” is: because we are unable to perform sufficiently detailed observations on a macroscopic scale.

\section{Persistence of Information and the Appearance of a Classical World}

While the decoherence argument is undoubtedly correct, I believe that its relying primarily on a practical limitation due to the macroscopic nature of the measuring apparatus (and of the observer) hides a philosophically more significant underlying fact. This is the fact that the crucial requirement for the equivalence of the quantum superposition and the corresponding classical mixture is the persistence of information about the value of the observed property (Sabbadini, 2006). But persistence of information is a general characteristic of all experiencing by an embodied observer: therefore an embodied observer must necessarily experience the world as classic.

This statement needs to be more carefully analyzed. A general observation performed on the von Neumann chain $\mathbf{S}+\mathbf{M}_{1}+\mathbf{M}_{2}+\ldots+\mathbf{M}$ consists of the simultaneous measurement of a property of each system involved, i.e. the measurement of a property $Q$ of $\mathbf{S}$, a property $R_{1}$ of $\mathbf{M}_{1}$, a property $R_{2}$ of $\mathbf{M}_{2}, \ldots$, a property $R$ of $\mathbf{M}^{8}$. Now, the persistence of information theorem states the following: the superposition which is the outcome of a measurement process and the corresponding classical mixture are exactly equivalent (i.e. the interference terms vanish exactly) for predicting the results of all observations performed on the total system $\mathbf{S}+\mathbf{M}_{1}+\mathbf{M}_{2}+\ldots+\mathbf{M}$, except those in which all the properties $Q, R_{1}, R_{2}, \ldots, R$ are incompatible with the corresponding measurement observables $\mathrm{P}, \mathrm{M}_{1}, \mathrm{M}_{2}, \ldots, \mathrm{M}^{9}$. But it is precisely in the value of these observables that information about the value of the observed property $\mathrm{P}$ is stored ${ }^{10}$. The performance of the simultaneous measurement of $\mathrm{Q}, \mathrm{R}_{1}, \mathrm{R}_{2}, \ldots$, $\mathrm{R}$ alters the state of all the systems in the von Neumann chain in a way that destroys all information about the value of the observed property $\mathrm{P}$.

When such is the case, we should not expect the superposition and the mixture to be equivalent. In fact in the quantum eraser experiments we have a concrete example of a situation closely parallel to the one just described, in which the interference terms do not vanish and the superposition and the mixture are not equivalent (see Appendix). On the other hand, if at least one of the properties $\mathrm{Q}, \mathrm{R}_{1}, \mathrm{R}_{2}, \ldots, \mathrm{R}$ is compatible with the corresponding measurement observable, say if $R_{1}$ is compatible with $M_{1}^{11}$, the information about the value of the observed property $\mathrm{P}$ is not lost: it keeps being stored in the system $\mathbf{M}_{\mathbf{1}}$.

A couple of observations are in order at this point. First, ordinarily we are only interested in measurement processes in which information about the value of whatever is measured persists. A measurement process that leaves no trace of the value that is measured we would not consider a "proper measurement" (It would rather belong with the quantum eraser experiments, see Appendix). Therefore, although in principle the outcome of a measurement process is always represented by a quantum superposition, for proper measurements we are free to replace the quantum superposition with the corresponding classical mixture. There is actually no "collapse of the state vector"-but, within the frame of proper measurements, it is just as if it did exist.

Second, we are ordinarily interested in measurement processes in which information gets amplified to a macroscopic scale. The von Neumann chain then includes at least a macroscopic system, and performing an observation of a property $\mathrm{R}$ incompatible with the measurement observable $\mathrm{M}$ of the macroscopic system is not realistically feasible. It would require knowing the exact quantum state of the macroscopic system and being able to act precisely on it.

Furthermore the von Neumann chain generated by a measurement process extends well beyond the measuring apparatus: it includes the laboratory environment, whose quantum state comes to be correlated with the state of the measuring apparatus, any observer that might be present-or even might later read the outcome of the measurement, etc. In principle the correlations spread through the whole universe in the great entangled totality of

\footnotetext{
${ }^{8}$ If no measurement is performed on a system, say $\mathbf{M}_{1}$, the the corresponding property is the identity $\mathrm{I}_{1}$ in the Hilbert space of $\mathbf{M}_{\mathbf{1}}$.

${ }^{9}$ In mathematical notation, $[\mathrm{Q}, \mathrm{P}] \neq 0,\left[\mathrm{R}_{1}, \mathrm{M}_{1}\right] \neq 0,\left[\mathrm{R}_{2}, \mathrm{M}_{2}\right] \neq 0, \ldots,[\mathrm{R}, \mathrm{M}] \neq 0$.

${ }^{10}$ “Information" is here taken to mean "potentially retievable information". Therefore the object system itself contains information about the value of its property P. Likewise, at the end of the measurement process, any of the systems in the von Neumann chain contains information about the value of $\mathrm{P}$ in the object system.

${ }^{11}$ In mathematical notation $\left[\mathrm{R}_{1}, \mathrm{M}_{1}\right]=0$.
} 
everything. The idea of performing the simultaneous measurement of properties incompatible with the measurement observables on all the systems involved becomes increasingly unthinkable as the chain extends farther afield. From this point of view the persistence of information approach rejoins the standard macroscopic decoherence arguments.

\section{Is the World Objective?}

Although in a measurement process the information is stored in the whole von Neumann chain of interacting systems, i.e. a measurement process leaves many traces in the world, there is one trace that is philosophically of paramount importance to us: that is the information that reaches an embodied consciousness (an "observer”) and gets stored in the observer's body (e.g. by causing a specific firing pattern in her or his brain).

In this context we should think of the body of the observer as a quantum system included in the von Neumann chain of the measurement process. Now, if we enlarge our vision and look at quantum measurement as a general pattern of all processes by which an embodied consciousness acquires information, we are led to view the body as a quantum system constantly linked to its environment by innumerable von Neumann chains producing in it an ongoing stream of changes correlated with whatever is happening in the environment ${ }^{12}$. A trace of the happening persists in the observer's body as long as there is in it a subsystem (not necessarily localized) whose state is correlated with the outer event ${ }^{13}$. Our experience of the world consists of the constant accumulation and transformation of such traces. But this fact is precisely what hides the quantum nature of reality from our direct perception and causes the world to appear objective, i.e. possessing well defined properties and obeying a Boolean either/or logic.

The classical appearance of the world is not exclusively linked with the traces it creates in our bodies. The decisive factor is the persistence of potentially retrievable information anywhere, and therefore primarily in the world itself. But, no matter where else the information may be stored, it has to be stored in our own body for us to have an experience. This is significant. It implies that the apparent objectivity of the world is intrinsic to the process of experiencing. For an embodied consciousness experiencing necessarily means experiencing an objective world.

From the very beginning of quantum physics some scientists pointed out that quantum physics is not a closed description of an objective world. The theory refers to subjectivity in an essential way: its basic postulates cannot be formulated without explicitly mentioning the process of observation. Furthermore, it was later realized that the theory is actually incompatible with the ordinary notion of objects, things, as localized systems endowed with objective properties (Bell, 1988). The classic Cartesian separation of mind and matter is certainly an inadequate frame for understanding quantum reality. But the naive notion of subjectivity directly affecting material reality (being responsible for the collapse of the state vector) is not much more satisfactory. I would like to propose that the relationship between subject and object suggested by quantum physics is subtler than that, i.e. that subject and object are co-arising manifestations of a common matrix of experiencing.

The philosophical stance most compatible with quantum physics then is neither naive realism nor pure idealism, but a phenomenological position that assumes the notion of experiencing as primary. In the act of experiencing we experience ourselves as subjects and the world as object.

\section{Conclusion}

When we give up the primacy of the objective world all our science must be understood not as a map of objective reality, of matter, but as mapping the intrinsic order of the process of experiencing. Quantum physics then is not, properly speaking, the mathematics of matter: it is the mathematics of experiencing.

If we assume experiencing to be the general matrix of reality and view subjectivity as an emergent aspect of it, then consciousness does not appear to be a localized phenomenon associated with special forms of organization of matter, as it is ordinarily viewed in the context of dualistic thinking. It appears to be a constitutive aspect of reality in general. There is no world without consciousness experiencing it, and there is no consciousness except experiencing a world.

\footnotetext{
${ }^{12}$ This description should be considered only a first approximation. In fact the body can be considered a definite subsystem of the universe only for a very short time and in a crude approximation. The circulation of information actually pervades the whole universe. The problem of understanding our existence as individual selves embodied in separate bodies is perhaps the most difficult philosophical problem.

${ }^{13}$ Such a state may persist until it gets erased by perturbations or by interacting with other bits of information generating further experiences. It would be interesting to speculate about how all this relates to memory and to our sense of time.
} 
If experiencing is the matrix of reality and is coextensive with the world and with consciousness, we implicitly open up to the possibility that experiencing, and therefore the experiencing consciousness and the experienced world, may assume many different forms, possibly some much larger or smaller or qualitatively different than our own. We obviously recognize as consciousness only a type of subjectivity that is close enough to our own. Admittedly we enter here a purely speculative realm: but it is good to remember that "there are more things in heaven and earth than are dreamt of in [our] philosophy ${ }^{14,}, .$.

Ancient and primitive people saw the whole world as animated, as having a soul (anima mundi). We have moved a long way from their vision and have been mesmerized by the notion of an objective, essentially mechanical world in which consciousness exists only in small islands (if at all, if it is not a mere epiphenomenon of matter). But, curiously, diving deep into the science of the objective, diving deep into the heart of matter, we may have reached the limit of our objectifying enterprise. Understanding the world's primary reality as experiencing, and therefore coextensive with consciousness, may be a small step towards rediscovering the world's soul.

\section{References}

Bell, J. S. (1988). Speakable and Unspeakable in Quantum Mechanics. Cambridge: Cambridge University Press.

Daneri, A., Loinger, A., \& Prosperi, G. M. (1962). Quantum Theory of Measurement and Ergodicity Conditions. Nuclear Physics, 33, 297-319. Reprinted in Wheeler, J. A., \& Zurek, W. H. (Eds.) (1983). Quantum Theory and Measurement (pp. 657-679). Princeton, NJ: Princeton University Press.

Everett, H. (1957). "Relative State” Formulation of Quantum Mechanics. Reviews of Modern Physics, 29, 454-462. Reprinted in Wheeler, J. A., \& Zurek, W. H. (Eds.) (1983). Quantum Theory and Measurement (pp. 315-323). Princeton, NJ: Princeton University Press.

Greenstein, G., \& Zajonc, A. (1997). The Quantum Challenge. Boston, MA: Jones \& Bartlett.

Jaynes, E., in Barut, A.O. (Ed.) (1980). Foundations of Radiation Theory and Quantum Electronics. New York: Plenum.

Joos, E. (Ed.) (2003). Decoherence and the Appearance of a Classical World in Quantum Theory. Berlin: Springer. http://dx.doi.org/10.1007/978-3-662-05328-7

Kim, Y., Yu, R., Kulik, S. P., Shih, Y. H., \& Scully, M. (2000). A Delayed Choice Quantum Eraser, Physical Review Letters, 84, 1-5.

Lanz, L., Prosperi, G. M., \& Sabbadini, A. (1971). Time Scales and the Problem of Measurement in Quantum Mechanics, Nuovo Cimento, 2B, 184-192. http://dx.doi.org/10.1007/BF02723082

Ou, Z. Y., Wang, L. J., Zou, X. Y., \& Mandel, L. (1990). Evidence for Phase Memory in Two-Photon down Conversion through Entanglement with the Vacuum. Physical Review A, 41, 566. http://dx.doi.org/10.1103/PhysRevA.41.566

Sabbadini, S. A. (2006). Persistence of Information in the Quantum Measurement Problem. Physics Essays, 19, $135-150$. http://dx.doi.org/10.4006/1.3025774

Schrödinger, E. (1935). Die gegenwärtige Situation in der Quantenmechanik. Naturwissenschaften, 23, 807-812, 823-828, 844-849. Reprinted in Wheeler, J. A., \& Zurek, W. H. (Eds.) (1983). Quantum Theory and Measurement (pp. 152-167). Princeton, NJ: Princeton University Press.

Scully, M. O., \& Druhl, K. (1982). Quantum Eraser: A Proposed Photon Correlation Experiment Concerning Observation and "Delayed Choice” in Quantum Mechanics. Physical Review A, 25, 2208. http://dx.doi.org/10.1103/PhysRevA.25.2208

von Neumann, J. (1932). Mathematical Foundations of Quantum Mechanics (pp. 347-445). Princeton, NJ: Princeton University Press. Reprinted in Wheeler, J. A., \& Zurek, W. H. (Eds.) (1983). Quantum Theory and Measurement (pp. 549-647). Princeton, NJ: Princeton University Press.

Wigner, E. (1967). Remarks on the Mind-Body Question. Originally Published in Good, I. J. (Ed.) (1961). The Scientist Speculates (pp. 284-302). London: Heinemann. Reprinted in Wheeler, J. A., \& Zurek, W. H. (Eds.) (1983). Quantum Theory and Measurement (pp. 168-181). Princeton, NJ: Princeton University Press.

\footnotetext{
${ }^{14}$ Hamlet (1.5.167-8).
} 


\section{Appendix-The Quantum Eraser}

The idea of a “quantum eraser” was originally proposed by Jaynes (Jaynes \& Barut, 1980) and independently by Scully and Druhl (Scully \& Druhl, 1982), and it has since been realized in numerous experiments with a variety of setups. Here it will be analyzed in the particularly clear form described by Greenstein and Zajonc (Greenstein \& Zajonc, 1997), based on the original suggestion by Scully and Druhl and the subsequent realization by Ou et al. (Ou, Wang, Zou, \& Mandel, 1990).

The basic setup is a Mach-Zehnder interferometer (Figure 1). A beam of incoming photons is split into beams 1 and 2 by a first beam splitter $\mathrm{BS}_{1}$, a phase shifter PS alters the phase of beam 2 by $\phi$, and the two beams are re-combined by a second beam splitter $\mathrm{BS}_{2}$. Detectors $\mathrm{D}_{1}$ and $\mathrm{D}_{2}$ reveal the outgoing photons. The incoming beam is of sufficiently low intensity, so that only one photon at a time is introduced in the apparatus. As the phase $\phi$ is varied, an interference pattern is observed in the counts of $D_{1}$ and $D_{2}$.

Now two non-linear down-conversion crystals, $\mathrm{X}_{1}$, and $\mathrm{X}_{2}$, are inserted on the photon's path. When the photon enters one of these crystals it is split into a pair: one member of the pair, which we shall call the primary photon, keeps on traveling through the interferometer, while the other, which we shall call the secondary photon, is detected by an additional counter, $d_{1}$ or $d_{2}$. The firing of $d_{1}$ or $d_{2}$ now provides which-path information about the primary photon, and the interference pattern disappears.

But the which-path information can be erased: if we place a third beam splitter $\mathrm{BS}_{3}$ at the intersection of the paths of the secondary photon from the down-conversion crystals, the firing of $d_{1}$ or $d_{2}$ no longer tells us which path the primary photon has taken. The experiment then shows that an interference pattern re-appears in the coincidence counts between any of the primary detectors $D_{1}$ and $D_{2}$ and any of the secondary detectors $d_{1}$ and $\mathrm{d}_{2}$.

We can look at the entanglement of the primary and secondary photon as a microscopic analogue of the entanglement of the object system and the macroscopic apparatus at the end of a measurement process. The observed property $\mathrm{P}$ corresponds to a which-path observable of the primary photon and the measurement recording observable $\mathrm{M}$ corresponds to a which-path observable of the secondary photon. It can then be shown (Sabbadini, 2006) that the interference pattern shows up when and only when we perform a joint observation on the entangled pair that is incompatible with both which-path observables, i.e. when we measure a quantity of the form $\mathrm{Q} \otimes \mathrm{R}$, with $[\mathrm{P}, \mathrm{Q}] \neq 0$ and $[\mathrm{M}, \mathrm{R}] \neq 0$. Such an observation erases all information about the previous "measurement”: in these circumstances the equivalence of the quantum superposition and the corresponding classical mixture does not hold, and we indeed expect an interference effect to show up.

It is interesting to notice that the quantum eraser can be performed as a "delayed choice” experiment and it has in fact been performed as such (Kim, Yu, Kulik, Shih et al., 2000). One can insert the beam splitter BS 3

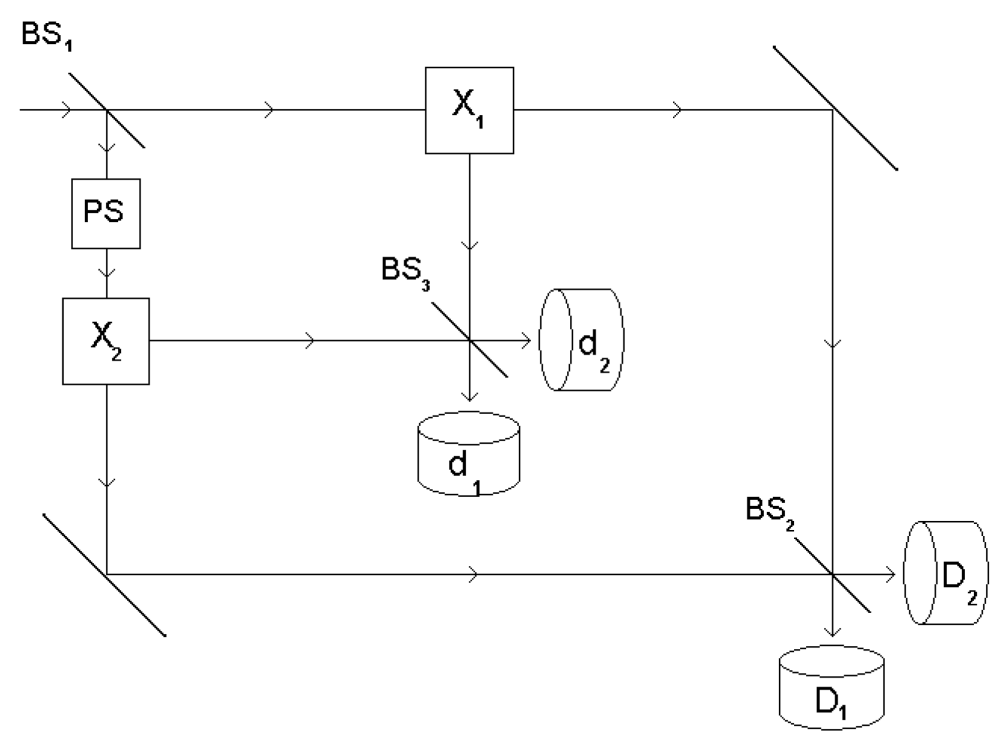

Figure 1. Schematic representation of the quantum eraser described by Greenstein and Zajonc. 
after the photon has already interacted with $\mathrm{BS}_{1}$, and even after it has already interacted with $\mathrm{X}_{1}$ and $\mathrm{X}_{2}$, thereby forcing it to choose between "going both ways" and "going either one way or the other" when it is already "well on its way". This peculiar feature of quantum mechanics has been the object of some discussion, and some people feel that it implies in some way "changing the past". No such doubt can arise in the "persistence of information" approach, in which inserting or not inserting the third beam splitter simply corresponds to performing different observations on the system. If the observation we choose to perform conserves information about the previous measurement, the equivalence proof will hold and an "either-or" situation will appear; otherwise it will not. 